\title{
Fabrication of Langmuir-Blodgett Film of Surface-Modified ZnO Nanoparticles Prepared by Solution Process
}

\author{
Yuhei Hasuike, Yoshitaka Kitamoto, Takeharu Tsuge, Masahiko Hara, Osamu Odawara, \\ Hiroyuki Wada* \\ Interdisciplinary Graduate School of Science and Engineering, Tokyo Institute of Technology, \\ 4259 Nagatsuta, Midori-ku, Yokohama 226-8502 Japan \\ Fax: +81 45924 5362, E-mail: wada.h.ac@m.titech.ac.jp
}

\begin{abstract}
$\mathrm{ZnO}$ nanoparticles were prepared by a solution method and a film of these nanoparticles was prepared by the Langmuir-Blodgett (LB) method. To prepare the LB film, the dispersibilities of $\mathrm{ZnO}$ nanoparticles in water and organic solvents were examined. The surface of the $\mathrm{ZnO}$ nanoparticles was modified by a surfactant, cetyltrimethylammonium bromide (CTAB), to improve their dispersibility. Using water as a solvent, dispersibility was improved by changing the $\mathrm{CTAB}$ concentration. However, aggregation of the nanoparticles occurred when they were transferred to chloroform to spread on water for fabrication of the LB film. With an organic solvent, aggregation of the $\mathrm{ZnO}$ nanoparticles in the LB film was improved when chloroform was used.
\end{abstract}

Key words: zinc oxide, nanoparticle, thin film, sol-gel method

\section{INTRODUCTION}

Recently, extensive studies on nanoparticles have been conducted owing to their unique properties $[1,2]$. Many potential applications of nanomaterials in research fields such as biotechnology, electronics, and energy have been proposed [3,4]. Among the various preparation methods of nanoparticles, solution process is the most promising and environmentally friendly.

Zinc oxide $(\mathrm{ZnO})$, a direct-transition wide-bandgap n-type semiconductor, is a widely studied material in this context. $\mathrm{ZnO}$ nanoparticles synthesized via the solution process have high crystallinity. They have found applications in light-emitting materials and electronic devices. Their thin films have found applications in gas sensors, photocatalysts, transparent conductive coatings, and light-emitting diodes [5]. For the utilization of $\mathrm{ZnO}$ in various fields, the preparation of thin films is important. The techniques for fabricating thin films are dip coating [6], spin coating [7], electrostatic spray coating [8], and the Langmuir-Blodgett (LB) method. Using the LB method, the potential for fabricating close-packed monolayer films on a substrate has been reported.

The theory of the LB method was established by Langmuir and developed by Blodgett. Typically, layers of an amphipathic surfactant are prepared on a substrate by this method. First, a dispersion of the surfactant in an organic solvent is spread on deionized (DI) water in a trough and a monolayer is formed on the surface of the water after reducing its area with a barrier. The monolayer is then transferred to the substrate. Similar attempts to prepare nanoparticle films rather than surfactant films have been reported [9-14]. An important prerequisite for this approach is that the nanoparticles should float on water. In general, because the surfaces of ceramic nanoparticles are hydrophilic, it is difficult to float them on water. Therefore, surface modification is used to change the surface properties. Usually, nanoparticles are likely to aggregate in accordance with Derjaguin, Landau, Verwey, and Overbeek theory. Improvements in this respect are important because aggregation degrades the properties of thin-film devices.

In this study, $\mathrm{ZnO}$ nanoparticles were prepared by a solution process and LB films of these nanoparticles were fabricated. Aggregation of the nanoparticles in the LB film was improved by surface modification of the $\mathrm{ZnO}$.

\section{EXPERIMENTAL}

$\mathrm{ZnO}$ nanoparticles were prepared by a normal solution process similar to that described in reference [15]. Zinc acetate dihydrate was dissolved in 2-propanol. The solution was vigorously stirred and a solution of sodium hydroxide in 2-propanol was added dropwise. The molar ratio of zinc acetate to sodium hydroxide was $1: 2$ in accordance with the following chemical equation. $\mathrm{Zn}\left(\mathrm{CH}_{3} \mathrm{COO}\right)_{2} \cdot 2 \mathrm{H}_{2} \mathrm{O}+2 \mathrm{NaOH}$

$$
\rightarrow \mathrm{ZnO}+2 \mathrm{CH}_{3} \mathrm{COONa}+\mathrm{H}_{2} \mathrm{O}
$$

The mixture was then aged for $3 \mathrm{~h}$.

After the preparation, the $\mathrm{ZnO}$ nanoparticles were separated by centrifugation and washed. They were then redispersed in various solvents, both aqueous and organic. For surface modification of the $\mathrm{ZnO}$ nanoparticles, cetyltrimethylammonium bromide (CTAB) was added to the dispersions. The nanoparticle dispersion was gently dropped onto water in a trough and LB films were fabricated on Si substrates. In the case of an organic solvent, the nanoparticle dispersion was directly spread on water. In the case of aqueous solvent, nanoparticles were extracted from the solvent and transferred to chloroform to spread them for the fabrication of LB films. LB films were fabricated by the following steps. The substrate was a $\mathrm{Si}$ wafer, which 
was treated with $2 \%$ hydrofluoric acid to obtain a hydrophobic surface and then washed with DI water and ethanol. The substrate was immersed in water in a trough. The nanoparticle dispersion $(15 \mathrm{~mL})$ was then spread on water and left for $5 \mathrm{~min}$. A barrier was moved at a rate of $0.83 \mathrm{~cm} / \mathrm{min}$ to reduce the surface area of the water from 600 to $100 \mathrm{~cm}^{2}$. The Si substrate was lifted at a rate of $3 \mathrm{~mm} / \mathrm{min}$.

The particle size of the prepared nanoparticles was measured by dynamic light scattering (DLS). The zeta potentials of the nanoparticles were investigated to understand the surface charge and surface modification. Absorbance spectra of the nanoparticle dispersions were measured on a spectrophotometer. The morphologies of the fabricated LB films were observed by a scanning electron microscope (SEM).

\section{RESULTS AND DISCUSSION}

The prepared nanoparticles were investigated. SEM images and X-ray diffraction patterns of the prepared nanoparticles were the same as those in our previous studies [16-18]. The particle size distribution is shown in Fig. 1. The average particle size was $7.7 \mathrm{~nm}$. Fig. 2 shows the zeta potential of the nanoparticles in solution at $\mathrm{pH}$ 9.42. The average value for the broad peak was $-16.2 \mathrm{mV}$. Therefore, the positively charged surfactant CTAB should be effective for surface modification of the negatively charged nanoparticles.

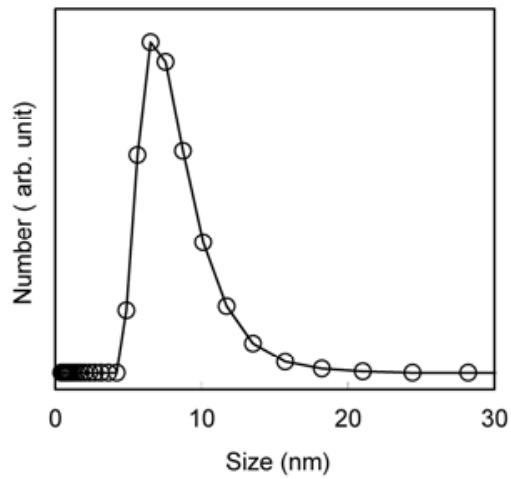

Fig. 1. Particle size distribution of synthesized $\mathrm{ZnO}$ nanoparticles.

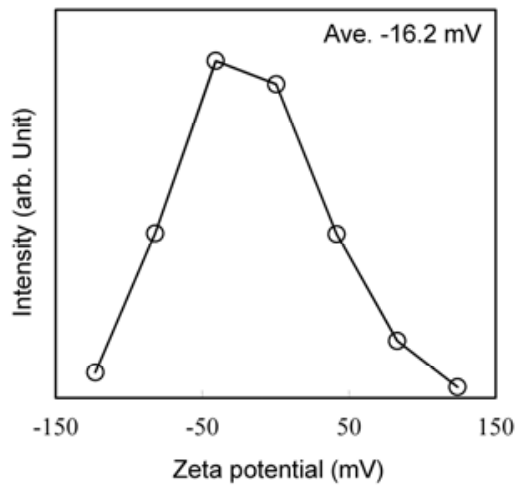

Fig. 2. Zeta potential distribution of synthesized $\mathrm{ZnO}$ nanoparticles.

In this study, two solvent systems were used to modify the surface of the $\mathrm{ZnO}$ nanoparticles. The first was an aqueous solvent system. For surface modification, surface electric charge is important. The effect of the $\mathrm{pH}$ of the aqueous solution on the surface electric charge was investigated. A powder of the washed $\mathrm{ZnO}$ nanoparticles was redispersed in the solution, the $\mathrm{pH}$ of which was then adjusted. Fig. 3 shows the zeta potentials of nanoparticles that were redispersed in DI water with $\mathrm{NaOH}$ at each $\mathrm{pH}$ value. An increase in $\mathrm{pH}$ decreased the zeta potential because the surface of the nanoparticles became electrically charged. Several mechanisms for electrical charging have been proposed, such as ionization of surface groups, isomorphous substitution, and specific ion adsorption [19]. In the case of $\mathrm{ZnO}$ nanoparticles, ionization of the surface groups is generally accepted. First, hydroxide groups are formed by adsorption of water molecules on the surface of the metal oxide [20]. Next, the surface is negatively charged by the following chemical equilibrium after the addition of base or positively charged after the addition of acid [21].

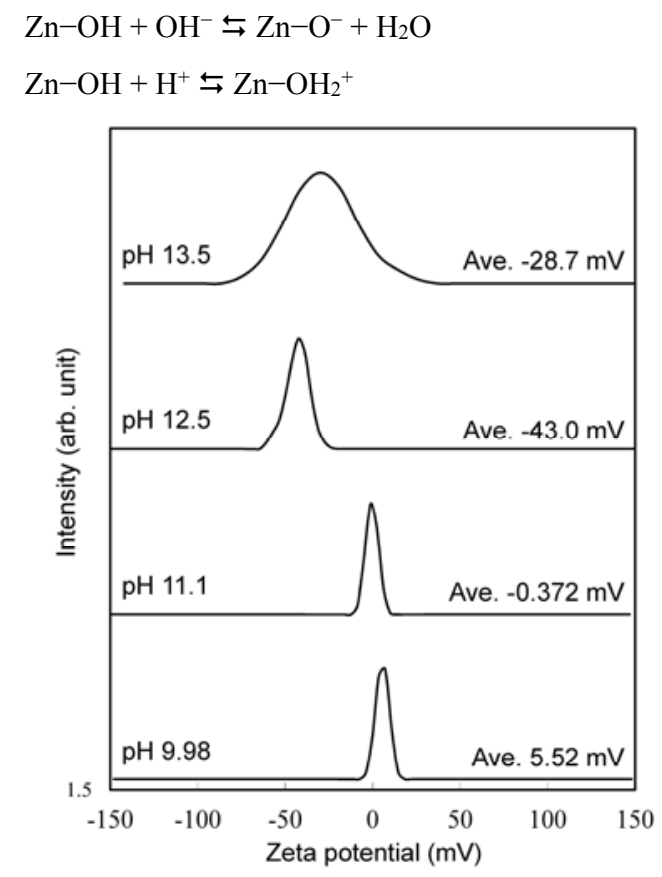

Fig. 3. Effect of $\mathrm{pH}$ on zeta potential of nanoparticles in aqueous solvent.

The point of zero charge (pzc), equivalent to the $\mathrm{pH}$ value at no electric charge, of this $\mathrm{ZnO}$ nanoparticle was approximately 11 . To modify the surface of the $\mathrm{ZnO}$ by a positively charged surfactant (CTAB), the $\mathrm{pH}$ must be higher than the pzc. To determine the $\mathrm{pH}$, the secondary particle sizes measured by DLS are shown in Fig. 4. These values are larger than that of $\mathrm{ZnO}$ without the addition of $\mathrm{NaOH}(7.7 \mathrm{~nm})$. There are two possible explanations for this: growth of the nanoparticles and their aggregation. According to SEM observations, the latter occurred at $\mathrm{pH}$ 12.5. Moreover, at $\mathrm{pH} 12.5$, precipitant was also observed because aggregation of the nanoparticles accelerated precipitation. In general, aggregation proceeds at around the pzc. Because the particle size at $\mathrm{pH} 13.5(31 \mathrm{~nm})$ was smaller than that at $\mathrm{pH} 12.5(120 \mathrm{~nm})$, the experimental conditions for further surface modification in this study were set at $\mathrm{pH}$ 13.5.

To modify the surface of the $\mathrm{ZnO}$ nanoparticles, 
CTAB was added to their dispersion at $\mathrm{pH}$ 13.5. Fig. 5 shows the zeta potential at each CTAB concentration. An increase in the CTAB concentration increased the zeta potential. The zeta potential was zero at $1.4 \mathrm{E}-5 \mathrm{M}$. Fig. 6 shows the secondary particle size as a function of $\mathrm{CTAB}$ concentration. The particle size was drastically increased at around $1.4 \mathrm{E}-5 \mathrm{M}$. In general, if the zeta potential is zero, the secondary particle size is increased by aggregation because of no repulsion by an electrostatic force. A reasonable mechanism would be that shown in Fig. 7. First, the positively charged CTAB surfactant is adsorbed through its hydrophilic head groups onto the negatively charged $\mathrm{ZnO}$ nanoparticles by electrostatic attraction as the CTAB concentration is increased up to $1.4 \mathrm{E}-5 \mathrm{M}(\mathrm{A} \rightarrow \mathrm{B})$ [22]. After full coverage by the surfactant, the zeta potential of the nanoparticles would be zero and their surface would be hydrophobic. The positively charged CTAB surfactant would then be absorbed on the nanoparticles through hydrophobic interaction of its tail $(\mathrm{B} \rightarrow \mathrm{C})$. Therefore, increasing the $\mathrm{CTAB}$ concentration continuously increased the zeta potential up to $+35.6 \mathrm{mV}$.

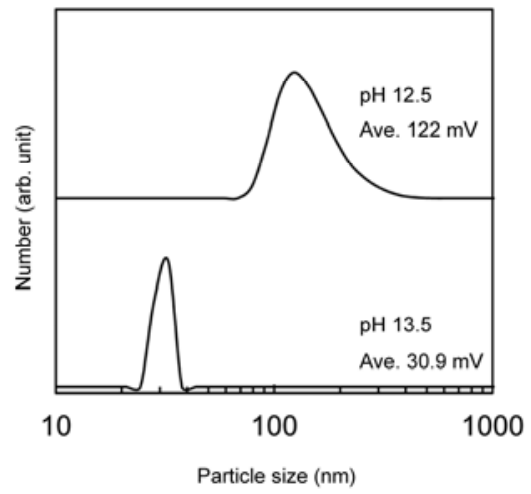

Fig. 4. The secondary particle size of $\mathrm{ZnO}$ nanoparticles at different $\mathrm{pH}$ values.

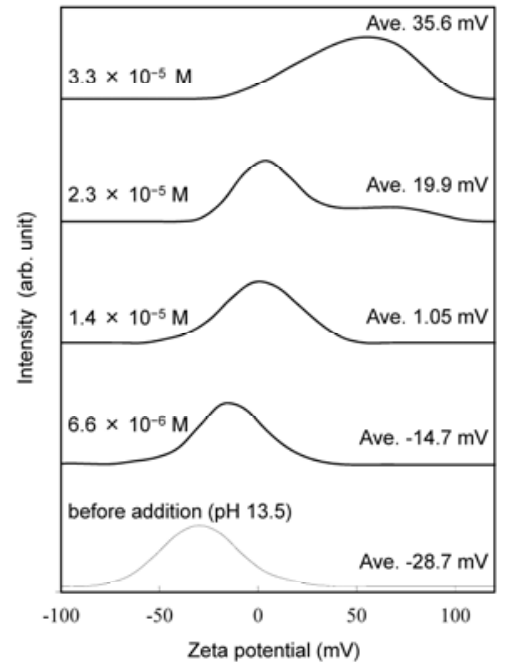

Fig. 5. Effect of $\mathrm{pH}$ on zeta potential of nanoparticles in aqueous solvent with CTAB.

Next, we examined organic solvent systems. Fig. 8 shows the effect of addition of CTAB on the zeta potential of nanoparticles in organic solvents. After washing, the $\mathrm{ZnO}$ nanoparticles were redispersed. In the case of hexane, as shown in Fig. 8A, the distribution of zeta potentials of the nanoparticles was not significantly changed by the addition of CTAB. In the case of chloroform, as shown in Fig. 8B, the distribution of zeta potentials of the nanoparticles was narrowed by the addition of CTAB. Surface modification of hydrophilic $\mathrm{ZnO}$ nanoparticles is important because without it they are not dispersed in organic solvents. If the surface is rendered hydrophobic by the modification, as shown in Fig. $7 \mathrm{~B}$, the $\mathrm{ZnO}$ nanoparticles can be dispersed in organic solvents. The modification is realized by adsorption of a surfactant onto the $\mathrm{ZnO}$ nanoparticles. To examine this hypothesis, absorption spectra of the supernatants of nanoparticle dispersions after the addition of CTAB were measured, as shown in Fig. 9. The absorption edge peak of $\mathrm{ZnO}$ nanoparticles, indicating the existence of nanoparticles in the supernatant, was not observed in the case of hexane (Fig. 9A), but it was observed for chloroform (Fig. 9B) The absence of the peak in hexane can be attributed to the precipitation of nanoparticles by aggregation. However, the $\mathrm{ZnO}$ nanoparticles would be capped by the surfactant in chloroform, forming a hydrophobic layer, and would thus be dispersed in organic solvents.

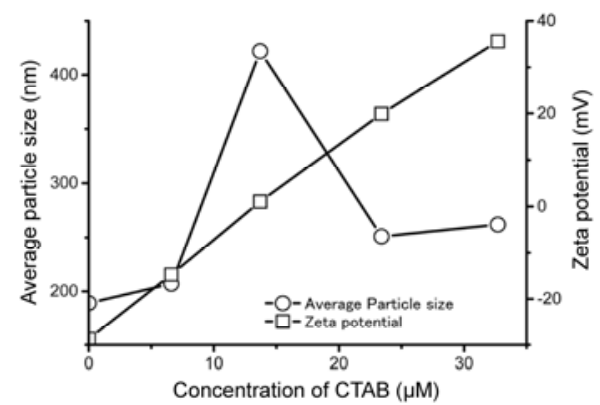

Fig. 6. Average particle size and zeta potential of $\mathrm{ZnO}$ nanoparticles as a function of CTAB concentration.

(A)

(B)

(C)

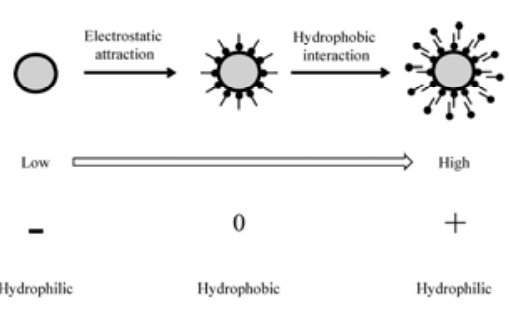

Fig. 7. Effect of CTAB concentration on surface charge and surface property of $\mathrm{ZnO}$ nanoparticles.

LB films were then fabricated using DI water and chloroform as solvents to disperse the nanoparticles. Fig. 10 shows SEM images of the fabricated LB films. In the case of an aqueous solvent (DI water), as shown in Fig. 10 A-B, the aggregation of the nanoparticles was observed. Surface modification to obtain a hydrophobic layer on the nanoparticles for the fabrication of LB films was successfully accomplished. However, aggregation would occur during extraction into chloroform. For the fabrication of LB films, it is necessary to float the $\mathrm{ZnO}$ nanoparticles on water by capping them with a hydrophobic layer. Simultaneously, the hydrophobic 
layer will promote aggregation of the nanoparticles in DI water. In the case of an organic solvent (chloroform), as shown in Fig. $10 \mathrm{C}-\mathrm{D}$, the aggregation in LB films was improved because the hydrophobic surface layer on the nanoparticles would promote their dispersal in such media and allow them to float on the water in the trough.
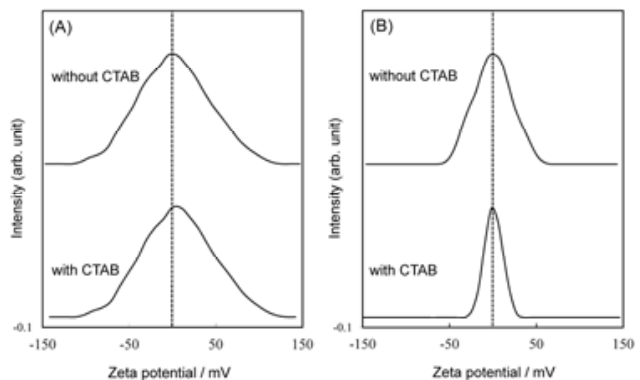

Fig. 8. Effect of CTAB on zeta potentials of $\mathrm{ZnO}$ nanoparticles in organic solvents: $(\mathrm{A})$ in hexane, (B) in chloroform.

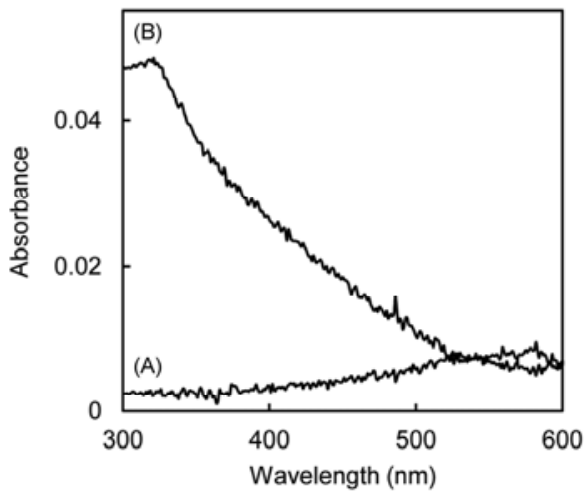

Fig. 9. Absorption spectra of supernatant of nanoparticle dispersions containing $\mathrm{CTAB}$ : (A) hexane, (B) chloroform.
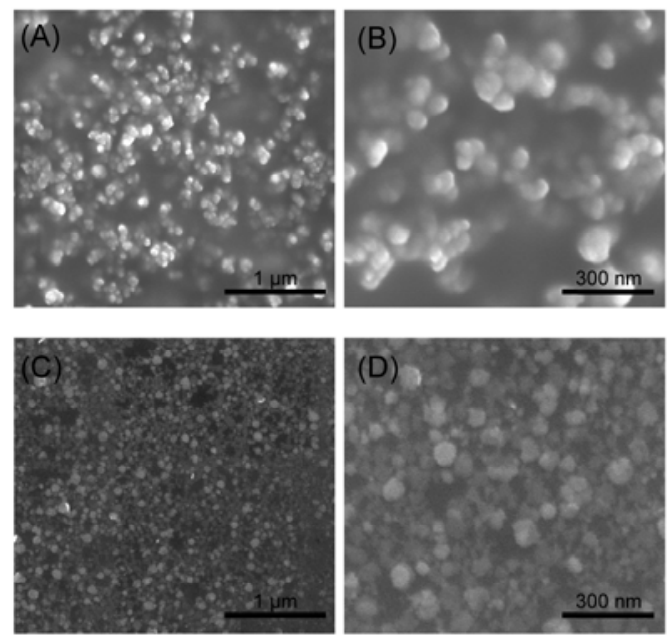

Fig. 10. SEM images of $\mathrm{ZnO}$ nanoparticle films: (A) and (B) aqueous solvent; (C) and (D) organic solvent.

\section{CONCLUSIONS}

$\mathrm{ZnO}$ nanoparticles have been capped with CTAB using chloroform as a solvent, and then an LB film of these nanoparticles was fabricated. The $\mathrm{ZnO}$ nanoparticles could be capped with the surfactant by changing the $\mathrm{pH}$ of the medium and the concentration of the surfactant. On increasing the CTAB concentration, a single capping monolayer was formed to render the surface of the nanoparticles hydrophobic, and then a bilayer was formed by hydrophobic interaction. CTAB-capped nanoparticles in DI water were likely to aggregate owing to their hydrophobic surface. LB films formed from them may be useful for optical and/or electrical devices such as gas sensors.

\section{REFERENCES}

[1] Al. L. Efros and A. L. Efros, Sov. Phys. Semicond. 16, 772 (1982).

[2] L. E. Brus, J. Chem. Phys. 80, 4403 (1984).

[3] M. Bruchez, M. Moronne, P. Gin, S. Weiss and A. P. Alivisatos, Science 281, 2013 (1998).

[4] W. C. W. Chan and S. Nie, Science, 281, 2016 (1998).

[5] Z. K. Tang, G. K. L. Wong, P. Yu, M. Kawasaki, A. Ohtomo, H. Koinuma and Y. Segawa, Appl. Phys. Lett. 72, 3270 (1998).

[6] Y. Lu, R. Ganguli, C. A. Drewien, M. T. Anderson, C. J. Brinker, W. Gong, Y. Guo, H. Soyez, B. Dunn, M. H. Huang, J. I. Zink, Nature, 389 (1997) 364.

[7] L. E. Scriven, Mat. Res. Soc. Symp. Proc. 121 (1988) 717.

[8] C. M.Ghimbeu, J. Schoonman, M. Lumbreras, M. Siadat, Appl. Surf. Sci. 253 (2007) 7483.

[9] S. Huang, K. Minami, H. Sakaue, S. Shingubara, T. Takahagi, Langmuir, 20 (2004) 2274.

[10] Y. Lu, G. L. Liu, L. P. Lee, Nano lett. 5 (2005) 5.

[11] J.-I. Park, W.-R. Lee, S.-S. Bae, Y. J. Kim, K.-H. Yoo, J. Cheon, S. Kim, J. Phys. Chem. B, 109 (2005) 13119.

[12] V. Aleksandrovic, D. Greshnykh, I. Randjelovic, A. Frömsdorf, A. Kornowski, S. V. Roth, C. Klinke, H. Weller, ACS Nano, 2 (2008) 1123.

[13] K. M. Gattás-Asfura, C. A. Constantine, M. J. Lynn, D. A. Thimann, X. Ji, R. M. Leblanc, J. Am. Chem. Soc. 127 (2005) 14640.

[14] L. Naszályi, A. Deák, E. Hild, A. Ayral, A. L. Kovács, Z. Hórvölgyi, Thin Solid Films, 515 (2006) 2587.

[15] L. Spanhel, M. A. Anderson, J. Am. Chem. Soc. 113 (1991) 2826.

[16] S. Tachikawa, A. Noguchi, T. Tsuge, M. Hara, O. Odawara, H. Wada, Materials, 4 (2011) 1132.

[17] S. Takahashi, Y. Hasuike, A. Noguchi, T. Tsuge, M. Ishikawa, O. Odawara, H. Wada, Jpn. J. Appl. Phys. 49 (2010) 052602.

[18] S. Takahashi, H. Wada, A. Noguchi, O. Odawara, Mater. Lett. 62 (2008) 3407.

[19] D. H. Everett, Basic principles of colloid science (Royal Society of Chemistry, London, 1988) p. 36.

[20] M. Nagao, J. Phys. Chem. 75 (1971) 3822.

[21] A.Degen, M. Kosec, J. Eur. Ceram. Soc. 20 (2000) 677.

[22] B. J. Ninness, D. W. Bousfield, C. P. Tripp, Colloids. Surf A: Physicochem. Eng. Aspects. 203 (2002) 21.

(Received January 30, 2015; Accepted July 24, 2015) 